\title{
Länger anhaltender Therapieerfolg
}

_ Für Patienten mit mittelschweren oder schweren Verläufen einer chronisch entzündlichen Darmerkrankung (CED) steht seit drei Jahren Vedolizumab (Entyvio $^{\circledR}$ ) zur Verfügung. Es ist indiziert, wenn konventionelle Therapieformen und TNF-a-Antagonisten nicht mehr helfen oder nicht vertragen werden. Neue Daten deuten darauf hin, dass der Integrin-An-

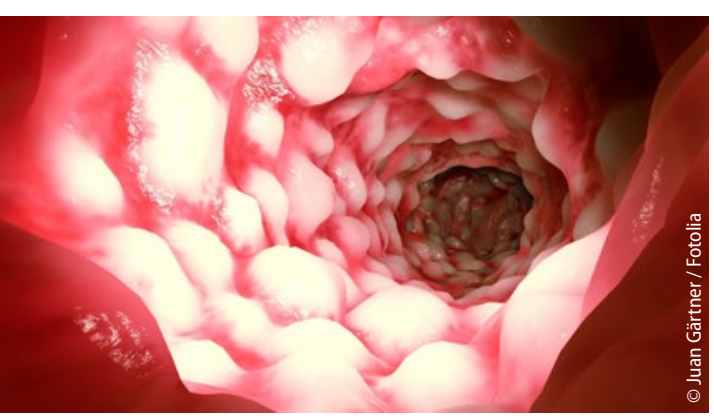

Eine schwere Form des Morbus Crohn. tagonist zur Remissionserhaltung in der Langzeittherapie effektiv und sicher ist.

„Der Wirkverlust beträgt in drei Jahren bei Colitis ulcerosa $9 \%$ und bei Morbus Crohn $17 \%$. Das ist weniger als bei anderen Therapieformenn, wobei es keine Head-to-head-Studien gibt“, berichtete Prof. Robert Ehehalt, Heidelberg.

Das Therapieziel bei M. Crohn und Colitis ulcerosa ist die Normalisierung der Lebensqualität, erreichbar durch eine anhaltende Remission und Mukosaheilung. Ca. 50\% der Patienten benötigen dazu eine Langzeittherapie, so Ehehalt.

Vedolizumab verhindert, dass Entzündungszellen in die Darmwand eindringen. Wenn der Patient auf das Medikament anspricht, dann spricht viel dafür, dass er gut damit leben kann.

Auch die mittelfristigen Daten zur Sicherheit und zu Nebenwirkungen stim- men hoffnungsvoll, ergänzte Prof. Jürgen Büning, Lübeck. Vedolizumab weist offensichtlich weniger Sicherheitsdefizite auf als TNF- $\alpha$-Inhibitoren oder Azathioprin. Das Infektionsrisiko sei nicht erhöht. Das Nebenwirkungsrisiko steigt weder mit der Therapiedauer noch mit dem Alter des Patienten an.

Eine frühere Studie hatte einen Hinweis auf ein erhöhtes Risiko für Wundheilungsstörungen unter der Therapie ergeben. Eine neuere Studie hat dieses Signal nicht bestätigt, sodass die Sorge, Patienten unter Vedolizumab zu operieren, gemildert ist, so Büning.

\section{Dr. Dirk Einecke}

- Pressekonferenz „Zulassung Vedolizumab (Entyvio ${ }^{\oplus}$ ) seit 2014 - 3 Jahre Vedolizumab (Entyvio ${ }^{\oplus) ! ", ~ 73 . ~ K o n g r e s s ~ d e r ~}$ Deutschen Gesellschaft für Gastroenterologie, Verdauungsund Stoffwechselkrankheiten; Dresden, September 2017 (Veranstalter: Takeda)

\section{Influenza-Schutz für die Generation 65+}

\section{Wirkverstärkter Impfstoff}

— Je älter die Menschen sind, desto niedriger sind die Antikörpertiter nach einer Impfung. Das Schlüsselwort dazu heißt Immunseneszenz, so Prof. Barbara Gärtner, Homburg/Saar.

Um den altersbedingten Veränderungen des Immunsystems Rechnung zu tragen, wurde bereits vor etwa 20 Jahren mit Fluad $^{\circledast}$ ein spezieller Impfstoff entwickelt. Der Impfstoff enthält einen Wirkverstärker, der die Immunantwort verbessern kann. „Wenn ich wirkverstärkt impfe, kommen deutlich mehr Makrophagen an den Ort der Impfung“, so Dr. Petra Sandow, Fachärztin für Allgemeinmedizin in Berlin. Mehr Makrophagen bedeuten mehr Antigen-präsentierende Zellen, damit mehr Immunzellen und im Endeffekt mehr Antikörper. Diese Antikörper setzen sich dann an das Hämagglutinin der Viren. Sandow:
"Je mehr unterschiedliche Antikörper ich habe, desto mehr kann ich diesen aggressiven Virus-Anteil blocken, der dafür verantwortlich ist, dass das Virus in die Zelle kommt. Das heißt: Die Anzahl der Antikörper ist enorm wichtig, um hier einen Effekt zu haben.“

Belegt wurde die Wirksamkeit des Impfstoffs unter anderem in der LIVEStudie mit über 170.000 mindestens 65-Jährigen in Norditalien in drei konsekutiven Grippesaisons der Jahre 2006 bis 2009. Bei mit Fluad ${ }^{\circledR}$ Geimpften lag die Rate an Influenza-bedingten Krankenhauseinweisungen im Vergleich mit einem herkömmlichen trivalenten Impfstoff um 25\% niedriger.

Christina Ott

- Pressekonferenz "Fluad - ein besonderer Impfstoff für einen besonderen Lebensabschnitt"; Frankfurt, August 2017 (Veranstalter: Seqirus)

\section{Prävention thrombovenöser Ereignisse bei Niereninsuffizienz}

Nierenfunktionsstörungen werden als Risikofaktor bei der medikamentösen Prophylaxe venöser Thromboembolien (VTE) bei internistischen, nicht-chirurgischen Patienten häufig nicht ausreichend beachtet, kritisierte Prof. Hanno Riess, Berlin, beim Kongress der International Society on Thrombosis and Haemostasis (ISTH). Die - oft älteren - Patienten haben ein erhöhtes Thromboseund Blutungsrisiko. Das niedermolekulare Heparin (NMH) Certoparin (Mono-Embolex ${ }^{\circledast}$ ) ist für diese Risikogruppe eine wichtige Option. Es kann mit Vorsicht auch bei schwerer Niereninsuffizienz (GFR $<30 \mathrm{ml}$ / min) eingesetzt werden.

Dies zeigte eine Subgruppenanalyse der Studie CERTIFY mit Patienten $>70$ Jahre mit schwer eingeschränkter Nierenfunktion (GFR $\leq 30 \mathrm{ml} / \mathrm{min}$ ). Certoparin war hinsichtlich der Häufigkeit thromboembolischer Ereignisse gegenüber UFH nicht unterlegen, aber signifikant sicherer.

Red.

- Nach Informationen von Aspen 